has been published ${ }^{8}$; it was calculated from evidence derived from the vegetation and from hunters' reports.

No research has been undertaken to determine whether dispersal in Now Zealand is largely independent of a population's density, or whether the rate fluctuates with changes in the population and its environment. Present work on sika deer, chamois, and thar should eventually answer this question.

Forest Research Institute,

New Zealand Forest Service, Rotorua, Now Zealand.

${ }^{1}$ Donne, T. E., The Game Animals of New Zealand (John Murray, London' 1924).

${ }^{2}$ Wodzicki, K. A., N.Z. Dept. Sci. Indust. Res. Bull., 98 (1950).

${ }^{3}$ Wodzicki, K. A., La Terre et la Vie, 1, 130 (1961).

'Riney, T., N.Z. Dom. Mus. Handbook, 4 (1955).

${ }^{5}$ Sweatman, G. K., and Williams, R. J., Trans. Roy. Soc. N.Z. (Zool.), 2 , $221(1962)$

в Riney, T., N.Z. J. Sci. Tech., B, 38, 1 (1956).

"Forbes, J., "New Zealand Deer Heads" (Country Life, London, 1924).

${ }^{8}$ Riney, T., Watson, J. S., Bassett, C., Turbott, E. G., and Howard, W.' E., N.Z. Dept. Sci.'Indust. Res. Bull., 135 (1959).

${ }^{9}$ Daniel, M. (personal communication).

\section{Significance of Allopreening in the Red Avadavat and its Development in Other Birds}

Alloprenning, that is, the habit of preening a neighbouring individual's feathers, is characteristic of many gregarious species of birds. Recent work on the Estrildine finch, the red avadavat (Amandava amandava), has shown that the amount of mutual grooming does not vary with the presence of peripheral dirt on the regions to which most of the allopreening is restricted, that is, the head. It thus seems likely that allopreening is more than simple co-operation in toilet behaviour between individuals which cannot effectively clean or arrange the feathers on their own heads as suggested by Morris ${ }^{1}$.

Preening is a subsidiary resting response, and in species in which the individuals are forced together, nibbling each others' feathers is a common activity, as in guillemots (Uria aalge) or young chickens (Gallus gallus). Birds seem to possess an innate response to feathers as primates have to fur ${ }^{2}$. Ritualized allopreening invitation postures are found in many highly gregarious 'contact' species and in 'contact' species where the members of a pair are closely associated throughout the year. In $A$. amandava there is specialized behaviour for eliciting grooming by a neighbour in the form of mandibulation nudging, and head. proffering.

When two red avadavats which are unfamiliar with each other approach and clump, the clumper is invariably pecked first; but in response to this, an allopreening invitation posture is taken up which results in an amelioration of the aggressive behaviour and the assumption of mutual preening ${ }^{3}$. Between strangers, then, allopreening serves to reduce aggression between the clumping individuals, but when they are fully familiar and their relation. ship is characterized by an absence of foar and aggression, allopreening serves to strengthen the social bonds between the birds, as suggested for social grooming in primates ${ }^{2}$.

The allopreening invitation posture is rarely used as a simple appeasement gesture in the face of an all-out attack in the manner suggested by Morris ${ }^{1}$, but is only given if the submissive individual is in strong conflict between approaching and fleeing, and is usually at the same time sexually attracted to the attacker. Otherwise, the immediate response to attack is flight.

J. H. Sparks

Dopartment of Zoology,

Queen Mary College,

University of London.

${ }^{1}$ Morris, D., Behaviour, 7, 33 (1956).

${ }^{2}$ Zuckerman, S., The Social Life of Monkeys and Apes (Kegan Paul, Trench, Trubner, Ĺtondon, 1932).

${ }^{3}$ Sparks, J. H., Birds Illustrated, 8, 48 (1962).

\section{Fertilization of Hamster Eggs in vitro}

Fertilization of rabbit eggs in vitro has been reported by many workers in recent years and Austin ${ }^{1}$ concluded that "success seems most likely to have been attained by Smith (1951), Dauzier et al. (1954), Dauzier and Thibault (1959), Thibault et al. (1954), Thibault and Dauzier (1960), Moricard (1954 a,b), and, particularly, Chang (1959)". So far as is known, no authentic successful experiments to fertilize rodent eggs in vitro have been reported ${ }^{1-4}$. This communication describes a repeatable technique to fertilize the eggs of the golden hamster (Mesocricetus auratus) in vitro, and the results obtained by this technique.

Female hamsters that came into heat between 6 p.m. and 9 p.m. were killed at 6-7 a.m. the next morning. Since most of the females ovulate about $8 \mathrm{~h}$ after the onset of heat ${ }^{5}$, these females were considered to have ovulated at about 2-5 a.m. Thus, the eggs were considered to be about 1-5 h after ovulation at the time of recovery. The oviducts of an animal were isolated, placed on a piece of filter paper, and most of the blood adhering to the oviducts was rubbed off. The oviducts were then put on the centre of a watch glass $(4 \mathrm{~cm}$ in diameter) and were immediately covered with warm $\left(30^{\circ}-37^{\circ} \mathrm{C}\right)$ mineral oil (Squibb). Under a dissecting microscope and on a warm stage, the distended portion of the ampulla was incised with needles and the eggs in cumulus oophorus were pressed out with some of the tubal fluid. The oviducts were discarded and blood and tissue debris, if any remained in the watch glass, were removed with a pipette. A small drop of sperm suspension, about 1-3 times the amount of the egg clot, was then added through the needle (25-27 gauge) of a tuberculin syringe to the eggs that were under the mineral oil. The eggs and the sperm suspension were thoroughly mixed with a needle. The watch glass was then kept in an incubator at $36^{\circ} \mathrm{C}$.

The sperm samples were collected either from the uterus of a female mated with proved male about $0.5 \mathrm{~h}$, or $4-5 \mathrm{~h}$, previously, or from the caudal epididymis of a fertile male. Usually, a drop of dense sperm mass was put on the centre of a watch glass (6 $\mathrm{cm}$ in diameter) and covered with $2-4 \mathrm{ml}$. of a solution containing 10 parts of tissue culture medium, ' $T C$ 199' (Difco Laboratories), 3 parts of $\mathbf{M} / 8$ glycine, and $100 \mathrm{units} / \mathrm{ml}$. of penicillin $G$. Under the microscope, the dispersion of sperms could be seen, the motility assessed to choose the best sample, and a suspension of desired concentration of sperms taken through the needle into a tuberculin syringe.

All the glassware and instruments were washed with double distilled water, sterilized, and kept at $30^{\circ}-37^{\circ} \mathrm{C}$ before and during use. Aseptic precautions were maintained during the whole operation.

Seven to ten hours after insemination, the eggs in the watch glass were transferred to a slide, compressed gently between a coverslip and the slide, and examined under a phase-contrast microscope for evidence of fertilization. The eggs were considered to be fertilized only when enlarged sperm heads or male pronuclei were found in the vitellus. Particular attention was directed to the tail of fertilizing sperm in or around the vitellus (Fig. 1). Occasionally, one or more sperms with intact (unswollen) heads were found in the perivitelline space or on the surface of the vitellus. Such sperms were recorded as supplementary sperms.

When 171 eggs from 21 females were inseminated with sperms recovered from the uterus of females mated 4-5 h previously, 113 (66 per cent) were fertilized. Of these fertilized eggs, 76 (67 per cent) were monospermic and 37 (33 per cent) were polyspermic (24 dispermic, 7 trispermic, 5 tetraspermic, and 1 pentospermic, as shown by the number of pronuclei and that of sperm tails in or around the vitellus). Seventeen monospermic eggs and 14 polyspermic eggs had 1-5 supplementary sperms. 